\title{
A Sustainable Model for Preventive Services in Rural Counties: The Healthier Together Study
}

\author{
Zsolt Nagykaldi, PhD, Dewey Scheid, MD, Yan D. Zhao, PhD, Bhawani Mishra, PhD, \\ and Tracy Greever-Rice, $P h D$
}

Objective: The Healthier Together study aimed to implement and evaluate a sustainable, rural community-based patient outreach model for preventive care provided through primary care practices located in 3 rural counties in Oklahoma. Community-based wellness coordinators (WCs) working with primary care practitioners, county health departments, local hospitals, and health information exchange (HIE) networks helped residents receive high-priority evidence-based preventive services.

Methods: The WCs used a wellness registry connected to electronic medical records and HIEs and called patients at the county level, based on primary care practitioner-preferred protocols. The registry flagged patient-level preventive care gaps, tracked outreach efforts, and documented the delivery of services throughout the community. Return on investment (ROI) in participating organizations was estimated by the study team.

Results: Forty-four of the 59 eligible clinician practices participated in the study. Two regional HIEs supplied periodic health data updates for 71,989 patients seen in the 3 implementation counties. A total of 45,862 outreach calls were made by 6 WCs, 100,896 high-priority recommendations were offered to patients based on care gaps and 14,043 additional services were delivered. of all the patients reached, only 1917 (4.2\%) were up to date on all prioritized services. Participating primary care practices significantly improved the delivery of preventive services (mean increase: $20.2 \%$ across 12 services; $P<.001$; range: $7 \%$ to $43 \%$ ) and realized a mean ROI of $68 \%$. Health systems that employed the WCs earned a mean revenue of $\$ 175,000$, realizing a $75 \%$ ROI for the outreach program.

Conclusions: Although health care is under-resourced and segmented in many rural counties, when stakeholder partnerships are established, they may be able to achieve and economically sustain communitywide health improvement by creating a win-win situation for all partners. ( $\mathrm{J}$ Am Board Fam Med 2020;33:698-706.)

Keywords: Chronic Disease, Electronic Health Records, Health Information Exchange, Oklahoma, Practice-Based Research, Preventive Health Services, Primary Health Care, Public Health, Registries, Rural Health

\section{Introduction}

Preventing premature death and disability is among the main goals of health care. Strategies to achieve this

This article was externally peer reviewed.

Submitted 1 October 2019; revised 4 February 2020; accepted 7 February 2020.

From the College of Medicine, Department of Family and Preventive Medicine, University of Oklahoma Health Sciences Center, Oklahoma City, OK (ZN, DS); College of Public Health, Department of Biostatistics and Epidemiology, University of Oklahoma Health Sciences Center, Oklahoma City, OK (YDZ); Institute of Public Policy, Harry S. Truman School of Public Affairs, University of Missouri, Columbia, MO (BM); School of Medicine, Center for Health Policy, University of Missouri, Columbia, MO (TGR).

Funding: Agency for Healthcare Research and Quality (AHRQ Grant No. 1R18HS023237).

Conflict of interest: None. goal include promoting wellness, preventing disease and injury, early detection of diseases by screening, and chronic disease management to prevent or delay complications. A number of evidence-based, costeffective preventive measures have been identified, some more cost effective than others. Optimizing the delivery/receipt of 5 key preventive measures would save about 100,000 lives a year in the United States. ${ }^{1}$

While primary care practitioners (PCPs) make improvements to deliver these key preventive

Corresponding author: Zsolt J. Nagykaldi, PhD, College of Medicine, Department of Family and Preventive Medicine, University of Oklahoma Health Sciences Center, $900 \mathrm{NE}$ 10th Street, Oklahoma City, OK 73104 (E-mail: znagykal@ ouhsc.edu). 
services, they continue to struggle to provide all needed services to patients who could benefit. ${ }^{1-4}$ Well-established reasons include a continuous expansion of competing care priorities, ${ }^{5}$ and an increasing amount of nonclinical work that burdens health care professionals on a daily basis. ${ }^{6}$ At the same time, hospitals and county health departments (CHDs), even in rural areas, have become more engaged in prevention due to various mandates and programs. However, many of these initiatives remain uncoordinated or misaligned across local communities.

Rural health disparities are evident throughout the United States. These include poorer health and lower quality health care compared with urban and suburban populations. ${ }^{7}$ Rural residents tend to be older, less affluent, less educated, and carry a higher disease burden, including smoking, obesity, hypertension, diabetes, and cardiovascular disease. ${ }^{8}$ Greater health care needs are coupled with less than half per-capita physician workforce compared with urban and suburban counties. ${ }^{9}$ It is clear that innovative health care models must be designed and implemented in rural America that can bring together rural health stakeholders and help integrate primary care and public health within a learning health system. ${ }^{10-14}$

Among the many components of preventive care, systematic patient outreach, which initiates the care process, is perhaps the least supported component structurally and financially. As a possible solution for establishing scalable preventive outreach, telephone-based reminders have been shown to be effective for increasing the receipt of preventive services. ${ }^{15,16}$ It is well established that the use of patient reminders increases screening rates for breast, cervical, and colorectal cancers, ${ }^{17}$ and computerized reminder systems are known to increase the delivery of preventive services in primary care ${ }^{18}$ and in hospitals. ${ }^{19-22}$ For example, we have demonstrated that a preventive services registry can increase the delivery and receipt of services by about $25 \%$ when integrated into well-resourced care settings. ${ }^{23}$ A community-based registry is a feasible option for small rural practices; however, it requires extra investment into dedicated registry operators and connecting electronic medical record (EHR) systems to a common, regional database either directly or through a health information exchange (HIE). In a past study, we have successfully connected a community-based preventive registry to 35 practices in a rural region of Oklahoma through an HIE service. ${ }^{24}$

In this 4-year study, we designed, implemented, and evaluated a sustainable, rural community-based patient outreach model to improve preventive care provided through primary care practices located in 3 rural counties in Oklahoma. Six wellness coordinators (WCs) working with 44 PCPs, 3 CHDs, 3 local hospitals, and 2 HIEs helped rural residents receive evidence-based preventive services while creating a ROI for rural health stakeholders.

\section{Methods}

\section{Settings, Participants, and Program Structure}

Collaborating with an academic study team, the predominant regional health systems located in the 3 implementation counties hired 6 full-time WCs from the local community who had experience with patient outreach in rural health settings (eg, nursing or health promotion staff, clinic receptionists). These systems also owned the regional hospitals, which aligned their business interests to invest into the outreach initiative. In 2 of the counties, the WCs were located in the county hospital, while in 1 county, they placed calls from a primary care clinic affiliated with the health system. Local partners agreed informally that the WCs work for and on behalf of the entire community and all primary care practices, regardless of their institutional affiliations.

To alleviate the financial risk of the initial experiment, the WCs' first-year salaries were paid by the Healthier Together grant that the investigators received from the Agency for Health Care Research and Quality. However, after the first year, the burden of WC support transitioned to the local health system, contingent on sufficient revenues generated from preventive services referred from participating practices. Each county's practice cohort received a practice facilitator $(\mathrm{PF})^{25,26}$ to help individual practices select and improve the delivery of 3 to 4 high-priority preventive services, create patient outreach protocols, and accelerate the implementation of improved care delivery processes. In addition, all county communities chose to focus on smoking cessation and physical activity improvement as shared care priorities while some practices chose to also improve the documentation of high-priority clinical measures linked to prevention. 
Figure 1. Healthier Together county partnership structure.

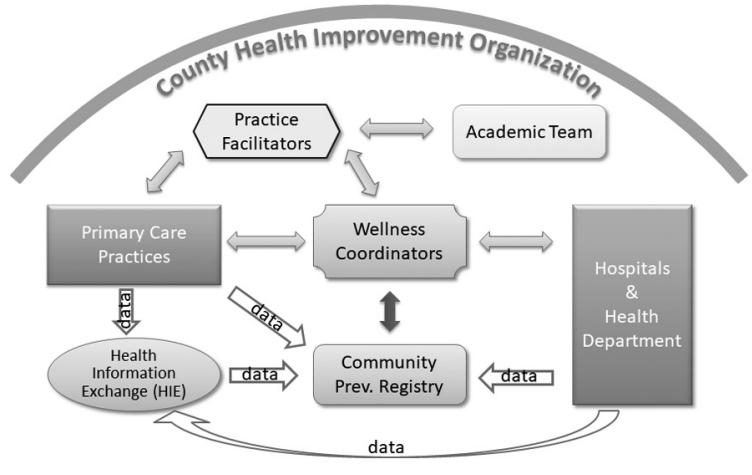

County collaborations were overseen by local nonprofit county health improvement organizations (CHIOs), health coalitions specific to Oklahoma, which acted as an "umbrella," to ensure transparency, create trust, and align the activities of disparate organizations with the goals of the community. Clinicians were engaged to participate in the project and provided ongoing feedback through their county champion representative on the CHIO board, in addition to constant contact with the PFs, who provided both implementation support and ongoing feedback to clinicians through practice visits. The main activity of participant clinicians consisted of the delivery of evidence-based preventive services and initiating referrals for a greater number of their patients whom they could not have engaged without the community-run outreach support. Two regional HIEs executed service contracts with the academic institution and also with health data generator organizations in the counties to supply interoperable clinical records (eg, Health Level Seven (HL7)-compliant Continuity of Care Documents) or data in other formats (eg, flat files) on county residents whose primary care home was in participating practices. Data on these individuals included not only information from the county, but also from other regional hospitals, diagnostic laboratories, imaging facilities, and specialty clinics. These data connections and the quality of data have evolved significantly in the course of the project. The CHDs were also engaged, albeit to a varying degree, and provided needed immunizations and facilitated the community health assessment process. A map of the county partnership structure is presented in Figure 1.

\section{Study Design}

We employed a quasi-experimental (pre-post) implementation study design to evaluate the effects of the Healthier Together program. Over a 3-year period, during staggered program implementation in each of the 3 counties, we computed annual baseline rates for delivering those services that were recommended for patients at the point of annual contact with the WCs. After a year of intervention, using the outreach registry and practice records, we then calculated the rates of service delivery including those patients who were contacted within the previous year, at least 3 months before the time of the calculation. We also determined the proportion of people who were up-to-date on all high-priority, practiceselected services at each measurement point. This process was then repeated annually.

\section{Data Sources}

\section{Outreach Calls and Preventive Services Delivery}

WC outreach calls were tracked systematically by a secure community registry, built on the Preventive Services Reminder System that the investigators developed earlier. ${ }^{23}$ The registry included a preventive care forecasting function that recommended person-tailored services based on HIE data, United States Preventive Services Task Force guidelines (A and $\mathrm{B}$ grades), personal risk factors, and seasonal variations of need. The WCs called county residents in their birth month in each consecutive project year, based on overlaps between personal care gaps flagged by the registry and a list of high-priority services selected by the practices and the local community working through the CHIO. Calls followed practice-tailored protocols and organizationapproved scripts with branching logic. Preventive service delivery was tracked by the Preventive Services Reminder System, practice EHRs, health system databases, and the HIEs.

\section{Econometric Data}

Financial data were collected through health care organization-specific and patient-level claims data and other appropriate cost and revenue sources (eg, medical chart documentation on service delivery) to conduct cost/revenue analyses. While most cost and revenue data were collected directly from health care providers in participating counties, 
when data were unavailable, estimates were derived from comparable local or regional data.

\section{Implementation Context}

We also received ongoing feedback from participating clinicians, hospital administrators, county health department directors, and other members of the CHIO Boards of Directors, according to domains proposed by Damschroder et $\mathrm{al},{ }^{27}$ which provided insights into implementation strategies, barriers and facilitators, participant perceptions, leadership engagement, organizational culture, regulatory climate, technology readiness, and successful implementation practices. The PFs took detailed field notes on observations pertaining to the implementation process in each practice and the study team maintained project-level progress notes on the development of the program. Stakeholder meeting agendas and minutes were also collected to document community-level discussions on the outreach program.

\section{Outcome Measures and Data Analyses}

Rates of preventive service delivery for selected services were calculated for a preimplementation baseline year and for the intervention year for each participating primary care practice using a combination of population-level HIE records and practice-level patient records and by generating representative samples of patients who were due for specific, United States Preventive Services Task Force-recommended services each year. Pre- and postimplementation service rates were compared as correlated proportions using the McNemar's test.

Return on investment (ROI) was defined at the health system level as the incremental revenue that was generated by the WCs, arising from extra referred preventive services compared with a baseline year, over the total cost of employing the WCs (approximately $\$ 50,000$ per WC per year). Practice-level ROI was defined as incremental practice revenue over the cost of extra services delivered compared with a baseline year. Health system revenues allowed practices to benefit from a shared patient outreach service, provided at the community level, at no cost to the practices. To conduct ROI analyses, we created an analytic database organized by intervention and provider type. Data collected across analytic dimensions were normed by prioritizing the use of common codes and billing data to calculate revenue as well as standard cost indicators, such as salaries and benefits, materials and supplies, and overhead for each priority preventive service. To establish a baseline, financial data were also collected for a year preceding the intervention in each practice and for each service. The discount rate for the base year (the "time value" of the money) was applied to intervention year projections and additional organizational investments into infrastructure were also considered. Incremental costs and revenues were calculated for the delivery of selected preventive services by organization and compared with those in the baseline year to calculate ROI estimates.

The study was approved and monitored by the University of Oklahoma Health Sciences Center Institutional Review Board.

\section{Results}

\section{High-Level Outcomes}

Between 2014 and 2018, 2 regional HIEs and onsite data extractions from electronic health records (EHRs) supplied periodic data updates for 71,989 patients, who were seen once or multiple times by participating practices during the study period. The demographic profile of the Healthier Together study population is shown in Table 1 and Table 2 .

On average, 6.94 evidence-based preventive care gaps were identified per person per contact by the community registry (ranging from 0 to 24 care gaps). Of these, typically, 2 to 3 recommendations were offered (suggested) by the WC over the phone per person per contact that overlapped with local practice or community health priorities. Across all participating practice sites and counties, over 100,000 high-priority preventive services were offered, more than 11,000 services were verbally accepted (agreed to be received) by patients at the time of contact, and over 14,000 additional services were delivered. Patients were then referred to their PCPs or other resources to receive accepted services. The most frequently accepted service recommendations are listed in Table 3.

\section{Care Delivery and Financial Outcomes}

A total of 45,862 outreach calls were made during the 4-year study and 100,896 high-priority preventive care recommendations were offered based on individual care gaps. The WCs spent 1146 hours on the phone with direct outreach communication. Of all the calls placed over the study period, patients 
Table 1. Demographics of the Healthier Together Study Population in Three Rural Oklahoma Counties between 2014 and 2018

\begin{tabular}{lc}
\hline $\begin{array}{l}\text { Demographic } \\
\text { Variables }\end{array}$ & Study Statistics ( $\mathrm{n}=71,989)$ \\
\hline Age, years & \\
Mean (SD) & $45.7(23.5)$ \\
Median & 46 \\
Range & 1 to 99 \\
Gender (female), \% & 57.0 \\
$\begin{array}{l}\text { Race/ethnicity } \\
\text { Medicare } \\
\text { beneficiaries }\end{array}$ & $\begin{array}{c}\text { Approximately } 70 \% \text { white* } \\
\text { Approximately 29\% of patients seen (10 } \\
\text { to 40\%) }\end{array}$ \\
\hline
\end{tabular}

*Race/ethnicity composition was estimated from local population statistics due to insufficient or inaccurate data in electronic medical records.

$\mathrm{SD}$, standard deviation.

were up to date on all identified services in 1917 contact instances $(4.2 \%)$. At the end of the first program implementation year, looking across all counties and the top 12 services, $20.2 \%$ (12.4 SD) more preventive services were delivered to patients compared with the corresponding baseline years, ranging from a $7 \%$ to a $43 \%$ increase in the delivery of services $(P<.001)$. The most significant improvements are summarized in Table 4 by service.

From increased service delivery compared with a year before, practices realized a mean ROI of $68 \%$ (a $68 \%$ revenue above offsetting the incremental cost of delivering more preventive care). This translated into $\$ 1800$ revenue per practice. The health systems that owned the county hospitals and employed the WCs earned, on average, $\$ 175,000$ in estimated revenues, compared with a baseline year, generating a $75 \%$ ROI (a 75\% revenue above offsetting the cost of employing the WCs). About 98\% of the hospitals' revenue came from increases in the provision of 3 referred screening tests, including colonoscopies, mammograms, and bone density scans. The other $2 \%$ of revenue came from
Table 3. Most Frequently Accepted Care Recommendations and Acceptance Rates at the Time of Wellness Coordinator Outreach in Three Rural Counties between 2014 and 2018

\begin{tabular}{lc}
\hline $\begin{array}{l}\text { Service Recommendations Patients } \\
\text { Accepted to Receive }(\mathrm{n}=11,607)\end{array}$ & $\begin{array}{c}\text { Percentage of All } \\
\text { Accepted }\end{array}$ \\
\hline Colon cancer screening & 16.03 \\
Adult dT-Tdap immunization & 11.14 \\
Influenza immunization & 10.01 \\
Mammography & 9.91 \\
Well-child visit & 6.55 \\
Zoster immunization & 6.46 \\
PAP smear & 5.99 \\
PCV13-Pneumo immunization & 5.52 \\
DEXA scan & 4.79 \\
Seatbelt use & 4.63 \\
Sun exposure advice & 2.42 \\
Smoking status documentation & 1.79 \\
Type 2 diabetes screening & 1.74 \\
HbA1c measurement & 1.62 \\
ACEI in diabetes & 1.53 \\
Lipid panel & 1.27 \\
MMR immunization & 1.1 \\
Hearing testing & 1.05 \\
Smoking counseling & 1.03 \\
VZV immunization & 0.89 \\
\hline
\end{tabular}

ACEI, angiotensin-converting enzyme inhibitor; DEXA, dualenergy X-ray absorptiometry; HbA1c, hemoglobin A1c; MMR, measles, mumps, rubella; PAP, Papanicolaou test; PCV13, pneumococcal conjugate vaccine 13 -valent; VZV, varicella zoster virus.

services provided by the outpatient clinics that were owned by the same health systems.

\section{Program Maintenance Outcomes}

Sustainability of the WC Outreach Program

Following the baseline year, 2 of the 3 county hospitals decided to sustain the outreach program by continuing funding for their WC positions. In one of these sustaining counties, the health system

Table 2. Geographic Locations and County Statistics of the Healthier Together Study Population in Three Rural Oklahoma Counties between 2014 and 2018

\begin{tabular}{lccc}
\hline County Locations & Total County Population & No. Clinics/No. Clinicians & No. Clinician Participants \\
\hline Central Oklahoma & 15,000 & $8 / 14$ & 11 \\
Northeast Oklahoma County 1 & 51,600 & $8 / 30$ & 23 \\
Northeast Oklahoma County 2 & 20,000 & $4 / 15$ & 10 \\
Totals & 86,600 & $20 / 59$ & 44 \\
\hline
\end{tabular}


Table 4. Overall Improvements in the Delivery of Preventive Services in Three Rural Counties, between 2015 and 2018

\begin{tabular}{lc}
\hline $\begin{array}{l}\text { Preventive Services Delivered } \\
(\mathrm{n}=14,043)\end{array}$ & $\begin{array}{c}\text { Improvement in } \\
\text { Delivery* }(\%)\end{array}$ \\
\hline Hypertension controlled & 43.0 \\
Diabetes checkup & 33.4 \\
HbAlc measurement & 33.0 \\
Smoking cessation counseling & 32.0 \\
Physical activity counseling & 23.0 \\
Well-child visit (EPSDT) & 19.0 \\
Mammography & 14.6 \\
Colonoscopy & 11.0 \\
BMI measurement & 10.8 \\
DEXA scan & 7.8 \\
HbAlc value documentation & 7.8 \\
Pneumococcal vaccination & 7.0 \\
\hline
\end{tabular}

BMI, body-mass index; DEXA, dual-energy X-ray absorptiometry; EPSDT, Early and Periodic Screening, Diagnostic and Treatment; HbA1c, hemoglobin A1c.

* Rates of preventive services that were received by patients after their annual contact with the wellness coordinators were computed across the three counties and over the project period. Improvement in the delivery of selected services was calculated by comparing a pre-implementation baseline year to rates at the end of the first implementation year for each primary care practice.

internalized the WC role after the first intervention year to benefit health system patients. This required establishing a parallel approach to maintain coverage for nonaffiliated patients. In the other sustaining county, which became the most successful project site, the local health system extended its support to 2 full-time equivalent (FTE) WCs after the first project year and continued supporting them, even beyond the end of the study. In the third project county, the wellness initiative was also on course toward sustainability; however, it deteriorated after the local health system was purchased by a large national entity, which changed the organization's care priorities during the second year of implementation.

\section{Fragility of Rural Health Care}

The number of major disruptive events in small, rural primary care practices was remarkably high, including disruptive ownership changes, EHR replacements, retirement or death of providers, loss and turnover of clinicians and key staff, and significant financial difficulties. Over half of the 44 participating practices experienced 1 or multiple major disruptive events during the 4-year study and 2 ceased to exist. For example, one practice was purchased by a large health system, thus causing multiple changes in key staff and switching to a new EHR. Later, the practice separated from the health system and lost electronic access to all accumulated patient data, which resulted in significant care disruptions and financial stress in the practice. These challenges caused considerable delays and difficulties in onboarding or sustaining practices in the project. They also hampered the ability of the practices to maintain a greater focus on the quality of care, as they were fighting for survival.

\section{Discussion}

To our knowledge, this study is one of the largest and most ambitious projects that attempted to create rural county-wide health improvement collaborations focusing on primary care with scalable outreach to entire rural communities and aimed at sustaining this activity in a cost-effective manner.

Despite numerous challenges, we have been able to implement broad county partnerships and functioning preventive outreach programs in each of the 3 rural counties. Two of these counties were able to maintain the program and pay for their WCs from revenues generated by the program after the first year of subsidized support. While, as expected, most of the financial returns on increased preventive services were realized by county hospitals run by local or regional health systems, 2 of the 3 hospitals have shown willingness to assume responsibility for investing into the outreach service, benefitting the local community, and 1 even extended the level of investment, doubling coordinator FTEs. Bringing under-resourced rural communities together to maintain shared resources that benefit all partners may be an alternative to the usual approach, whereby each organization or practice struggles to duplicate these services within their realms at a greater incremental cost to each. Supporting such resources collaboratively at the community level may also help diminish existing misalignments between investing much time and effort into initiating preventive services (mostly in primary care) and deriving more robust financial benefit from these services (mostly in imaging and screening facilities).

One of the most important underlying barriers to our project was the increasing structural fragility 
of rural primary care in Oklahoma over the past years. The level of major disruptive events in our study was almost identical to that in another study conducted concurrently in rural Oklahoma practices. ${ }^{28}$ Structural practice fragility affected the preventive outreach program in at least 3 major ways. First, the disappearance of autonomous, clinicianowned practices, which have been, historically, the most willing partners in rural primary care research and the diminishing viability of remaining small practices continued to narrow opportunities for introducing and testing much needed innovations in rural settings. Second, a new wave of practice buyouts by various health systems created increased commercial competition in small rural communities, further segmenting health care and disincentivizing community-level collaboration. Third, suboptimal investment into follow-up care resources locally (eg, advanced imaging, specialized therapy, counseling, or behavioral services) discouraged rural practitioners to more vigorously pursue preventive care in general. We argue that these challenges make innovative approaches to prevention even more urgently needed in rural communities.

There were several factors that made the implementation process difficult and contingent on certain infrastructural components and the timing of program deployment. Most importantly, creating a community-wide alignment of interests and buy-in across a diverse set of actors has been challenging, particularly between health care organizations that viewed themselves as competitors in the same region. In 2 counties, these organizations were reluctant to share WCs across their domains in the beginning and requested dedicated workforce they could use. One organization subsumed WC positions after the first implementation year, creating a health care gap in the county for patients unaffiliated with the specific health system that had to be bridged using alternative sources, including community grants the county received through the local $\mathrm{CHIO}$, which was enabled by their participation in Healthier Together. This suggests that rural communities, which are served overwhelmingly by a monolithic health system, may be more advantageously positioned to implement similar programs and may be able to create a better alignment between infrastructural investments and returns on these investments. This also suggests that rural communities without a willing, predominant health system may need to rely on external support (eg, by health extension services) to align their interests or establish alternative funding for health care innovations spanning across the community. This is especially relevant to the ownership of facilities that render referred screening services, including colonoscopy, mammography, and dual-energy X-ray absorptiometry (DEXA) scans as revenues from these services significantly surpass those earned from most others.

The second area of challenges included the establishment of health data interoperability between disparate health entities that included difficulties with patient data extraction, transmission, and load from electronic sources, as well as data cleaning, aggregation, and mapping. However, thorough this process, we have learned several key lessons that emerged from working with EHR and HIE vendors, including the following:

1. Real-world verification of the quality and timeliness of health information technology services is essential. Technology vendors may promise much but may deliver considerably less.

2. Technology contract specifics and pricing of services must be understood in detail, and clearly agreed upon in the beginning.

3. Data recipients should conduct scheduled, indepth data audits to verify the quality and comprehensiveness of information provided by the vendors.

4. Multiple contingencies must be developed for extraction, transmission, and load failures, which may happen more often than expected, including manual data extractions and chart reviews from clinical and billing records, working directly with data vendors, and tapping into other clinical data repositories.

A third area of challenges arose from difficulties with constructing and executing legal agreements with a variety of participant organizations in a timely manner. Legal uncertainties and unfamiliarity arose not only from the usual research aspects of the project, but the widely community-centered nature of the innovation. Large health systems, in particular, have shown some reluctance in agreeing to shared use of their data across multiple organizations. Additional business associate agreements and/or service contracts had to be executed with each organization. The academic partner and the HIEs have also executed data agreements and service contracts. These have often taken significantly longer time than expected. 
While the US public health system may be the ideal agent for promoting preventive health in rural communities, it is often not sufficiently resourced or not appropriately positioned to do so. Thus, our experiment to align rural health care stakeholders through an academic-led collaboration has produced important lessons regarding the feasibility and sustainability of alternative approaches. Our project generated a large amount of data that are being further analyzed at the time of this publication. Our findings will contribute to the development of a Guidebook that other organizations and community coalitions can use to replicate the project.

\section{Study Limitations}

Our study was limited to pre-post comparisons in each county, since using a separate control group or randomization were not feasible. This might have introduced baseline variation and time trends that we could not account for. However, we have captured a detailed and robust history of each implementation, which was leveraged to address some of these limitations. The study required the selection of rural counties that had certain resources and organizational structures in place, including a functioning community health coalition, a leading county hospital, and primary care practice/health system leadership willing to collaborate across the board. This may limit the generalizability of our study to rural counties that can establish these structures and deploy needed implementation components.

\section{Conclusions}

Although disparate health care entities in rural counties can be brought together to improve the health of the community through an organized wellness outreach program, the fragmentation and misalignment of the health care system makes it challenging to build such collaborations across the boundaries of organizations and their divergent interests. However, when these partnerships are established successfully, they may be able to achieve and economically sustain community-wide health improvement by creating a winwin situation for all partners.

The authors express their sincere appreciation to primary care practices, community partners, technology service providers, and patients, who participated in this study. Their support has been essential to the success of the community wellness program.
To see this article online, please go to: http://jabfm.org/content/ 33/5/698.full.

\section{References}

1. Sanchez E, ed. Preventive care: a national profile on use, disparities, and health benefits. Washington, DC: National Commission on Prevention Priorities; 2007.

2. Centers for Disease Control and Prevention. Surveillance summaries. Morbid Mortal Week Rep 2010;59:1-220.

3. McGlynn EA, Asch SM, Adams J, et al. The quality of health care delivered to adults in the United States. N Engl J Med 2003;348:2635-45.

4. Pham HH, Schrag D, Hargraves JL, Bach PB. Delivery of preventive services to older adults by primary care physicians. JAMA 2005;294:473-81.

5. Yarnall KS, Ostbye T, Krause KM, Pollak KI, Gradison M, Michener JL. Family physicians as team leaders: "time" to share the care. Prev Chronic Dis 2009;6:A59.

6. Christensen CM, Grossman JH, Hwang J. The innovator's prescription: a disruptive solution for health care. New York, NY: McGraw Hill; 2009.

7. Office of Rural Health Policy (ORHP). Rural Healthy People 2010_"Healthy People 2010: A companion document for rural areas." College Station, TX: Texas A\&M Health Sciences Center; 2010.

8. Bennett KJ, Bankole O, Probst JC. Health disparities: A rural-urban chartbook. Columbia, SC: South Carolina Rural Health Research and Policy Centers; 2008.

9. Oklahoma State University. State of the State's Rural Health. Stillwater, OK: Center for Health Sciences; 2008.

10. Institute of Medicine. Primary care and public health-Exploring integration to improve population health. Washington, DC: Institute of Medicine; 2012.

11. Felix K, Koo D, Dankwa-Mullan I, Miller T, Waalen J. Integration of primary care and public health. Am J Prev Med 2012;42(6).

12. The community as a learning system: Using local data to improve local health. Washington, DC: National Committee on Vital and Health Statistics; 2011.

13. Cantor J, Cohen L, Mikkelsen L, Panares R, Srikantharajah J, Valdovinos E. Community-centered health homes-Bridging the gap between health services and community prevention. Oakland, CA: Prevention Institute; 2011.

14. Folsom Group. Communities of solution: the Folsom report revisited. Ann Fam Med 2012;10:250-60.

15. Dietrich AJ, Tobin JN, Robinson CM, et al. Telephone outreach to increase colon cancer screening in Medicaid managed care organizations: 
a randomized controlled trial. Ann Fam Med 2013;11:335-43.

16. Eakin EG, Lawler SP, Vandelanotte C, Owen N. Telephone interventions for physical activity and dietary behavior change: a systematic review. Am J Prev Med 2007;32:419-34.

17. Zaza S, Briss PA, Harris KW, eds. The guide to community preventive services. New York, NY: Oxford University Press; 2005.

18. Dexheimer JW, Talbot TR, Sanders DL, Rosenbloom ST, Aronsky D. Prompting clinicians about preventive care measures: a systematic review of randomized controlled trials. J Am Med Inform Assoc 2008; 15:311-20.

19. Dexter PR, Perkins S, Overhage JM, Maharry K, Kohler RB, McDonald CJ. A computerized reminder system to increase the use of preventive care for hospitalized patients. N Engl J Med 2001;345: 965-70.

20. Hulscher ME, Wensing M, Grol RP, van der Weijden T, van Weel C. Interventions to improve the delivery of preventive services in primary care. Am J Public Health 1999;89:737-46.

21. Hunt DL, Haynes RB, Hanna SE, Smith K. Effects of computer-based clinical decision support systems on physician performance and patient outcomes: a systematic review. JAMA 1998;280:1339-46.

22. Kawamoto K, Houlihan CA, Balas EA, Lobach DF. Improving clinical practice using clinical decision support systems: a systematic review of trials to identify features critical to success. BMJ 2005; 330:765.

23. Nagykaldi Z, Mold JW. The role of health information technology in the translation of research into practice: an Oklahoma Physicians Resource/ Research Network (OKPRN) study. J Am Board Fam Med 2007;20:188-95.

24. Scheid D, Yeaman B, Nagykaldi Z, Mold JW. Regional health edecisions: A guide to connecting health information exchange in primary care. Oklahoma City/Norman, OK: Department of Family \& Preventive Medicine/Norman Physician Hospital Organization; 2013.

25. Nagykaldi Z, Mold JW, Aspy CB. Practice facilitators: a review of the literature. Fam Med 2005;37:581-8.

26. Nagykaldi Z, Mold JW, Robinson A, Niebauer L, Ford A. Practice facilitators and practice-based research networks. J Am Board Fam Med 2006;19: 506-10.

27. Damschroder LJ, Aron DC, Keith RE, Kirsh SR, Alexander JA, Lowery JC. Fostering implementation of health services research findings into practice: a consolidated framework for advancing implementation science. Implementation Sci 2009; 4:50.

28. Mold JW, Walsh M, Chou AF, Homco JB. The alarming rate of major disruptive events in primary care practices in Oklahoma. Ann Fam Med 2018;16:S52-S57. 